\title{
Erratum
}

\section{Erratum to: Classification of non-Riemannian doubled-yet-gauged spacetime}

\author{
Kevin Morand ${ }^{1,2, a}$, Jeong-Hyuck Park ${ }^{3,4, b}$ \\ ${ }^{1}$ Departamento de Ciencias Físicas, Universidad Andres Bello, Republica 220, Santiago de Chile, Chile \\ ${ }^{2}$ Departamento de Física, Universidad Técnica Federico Santa María, Centro Científico-Tecnológico de Valparaíso, Casilla 110-V, Valparaíso, \\ Chile \\ ${ }^{3}$ Department of Physics, Sogang University, 35 Baekbeom-ro, Mapo-gu, Seoul 04107, Korea \\ ${ }^{4}$ Center for Theoretical Physics of the Universe, Institute for Basic Science (IBS), Seoul 08826, Korea
}

Received: 14 October 2018 / Accepted: 26 October 2018 / Published online: 8 November 2018

(C) The Author(s) 2018

\section{Erratum to:}

Eur. Phys. J. C (2017) 77:685

https://doi.org/10.1140/epjc/s10052-017-5257-z

Equation (2.51) in the original article as well as the arXiv versions [v1, v2, v3] contains sign errors. It should read

$$
\begin{aligned}
Y_{i}^{\mu} \longmapsto & Y_{i}^{\mu}+H^{\mu \nu} V_{\nu i}, \\
\bar{Y}_{\bar{l}}^{\mu} \longmapsto & \bar{Y}_{\bar{l}}^{\mu}+H^{\mu \nu} \bar{V}_{\nu \bar{l}}, \\
k_{\mu}{ }^{a} \longmapsto & k_{\mu}^{a}-X_{\mu}^{i} \eta^{a b} h_{b}{ }^{\nu} V_{\nu i}-\bar{X}_{\mu}^{\bar{c}} \eta^{a b} h_{b}{ }^{\nu} \bar{V}_{\nu \bar{l}}, \\
\bar{k}_{\mu}{ }^{\bar{a}} \longmapsto & \bar{k}_{\mu}{ }^{\bar{a}}+X_{\mu}^{i} \bar{\eta}^{\bar{a} \bar{b}} \bar{h}_{\bar{b}}{ }^{\nu} V_{\nu i}+\bar{X}_{\mu}^{\bar{i}} \bar{\eta}^{\bar{a} \bar{b}} \bar{h}_{\bar{b}}{ }^{\nu} \bar{V}_{\nu \bar{l}}, \\
B_{\mu \nu} \longmapsto & B_{\mu \nu}-2 X_{[\mu}^{i} V_{\nu] i}+2 \bar{X}_{[\mu}^{\bar{i}} \bar{V}_{\nu] \bar{l}} \\
& +2 X_{[\mu}^{i} \bar{X}_{\nu]}^{\bar{l}}\left(Y_{i}^{\rho} \bar{V}_{\rho \bar{l}}+\bar{Y}_{\bar{l}}^{\rho} V_{\rho i}+V_{\rho i} H^{\rho \sigma} \bar{V}_{\sigma \bar{l}}\right) .
\end{aligned}
$$

Under these transformations, the DFT-vielbeins are invariant up to twofold local Lorentz rotations.

K. Morand, J.H. Park, Classification of non-Riemannian doubled-yet-gauged spacetime. Eur. Phys. J. C 77(10), 685 (2017). https://doi.org/10.1140/epjc/s10052-017-5257-z. arXiv:1707.03713 [hep-th]

Open Access This article is distributed under the terms of the Creative Commons Attribution 4.0 International License (http://creativecomm ons.org/licenses/by/4.0/), which permits unrestricted use, distribution, and reproduction in any medium, provided you give appropriate credit to the original author(s) and the source, provide a link to the Creative Commons license, and indicate if changes were made. Funded by SCOAP ${ }^{3}$.

The original article can be found online at https://doi.org/10.1140/ epjc/s10052-017-5257-z.

${ }^{a}$ e-mail: kevin.morand@1mpt.univ-tours.fr

b e-mail: park@sogang.ac.kr 